\title{
Charged-Particle Decay and Suppression of Primordial Power on Small Scales
}

\author{
Kris Sigurdson* and Marc Kamionkowski ${ }^{\dagger}$ \\ California Institute of Technology, Mail Code 130-33, Pasadena, California 91125, USA
}

(Received 20 November 2003; published 27 April 2004)

\begin{abstract}
We study the suppression of the small-scale power spectrum due to the decay of charged matter to dark matter prior to recombination. Prior to decay, the charged particles couple to the photon-baryon fluid and participate in its acoustic oscillations. However, after these charged particles decay to neutral dark matter, the photon-baryon fluid is coupled only gravitationally to the newly created dark matter. This generically leads to suppression of power on length scales that enter the horizon prior to decay. For decay times of $\sim 3.5 \mathrm{yr}$ this leads to suppression of power on subgalactic scales, bringing the observed number of galactic substructures in line with observation. Decay times of a few years are possible if the dark matter is purely gravitationally interacting, such as the gravitino in supersymmetric models or a massive Kaluza-Klein graviton in models with universal extra dimensions.
\end{abstract}

DOI: 10.1103/PhysRevLett.92.171302

PACS numbers: 95.35.+d, 98.35.Gi, 98.80.Cq

The standard inflation-inspired cosmological model, with its nearly scale-invariant power spectrum of primordial perturbations, is in remarkable agreement with observation. It predicts correctly the detailed pattern of temperature anisotropies in the cosmic microwave background $(\mathrm{CMB})[1]$, and accurately describes the large scale clustering of matter in the Universe [2]. However, on subgalactic scales there are possible problems with the standard cosmology that warrant further investigation. Namely, the model overpredicts the number of subgalactic halos by an order of magnitude compared to the 11 observed dwarf satellite galaxies of the Milky Way [3]. Several possible resolutions have been proposed to this apparent discrepancy, ranging from astrophysical mechanisms that suppress dwarf-galaxy formation in subgalactic halos (see, for example, Ref. [4]) to features in the inflaton potential that suppress small-scale power and thus reduce the predicted number of subgalactic halos [5].

In this Letter, we show that, if dark matter is produced by the out-of-equilibrium decay of a long-lived charged particle, then power will be suppressed on scales smaller than the horizon at the decay epoch. Unlike some other recent proposals, which suppress small-scale power by modifying the dark-matter particle properties [6], ours modifies the dark-matter production mechanism. In the model we discuss here, prior to decay the charged particles couple electromagnetically to the primordial plasma and participate in its acoustic oscillations. After decay, the photon-baryon fluid is coupled only gravitationally to the neutral dark matter. This generically leads to suppression of power for scales that enter the horizon prior to decay. This suppression reduces the amount of halo substructure on galactic scales while preserving the successes of the standard hierarchical-clustering paradigm on larger scales. Apart from the changes to the model due to the decay process, we adopt the standard flat-geometry $\Lambda \mathrm{CDM}$ cosmological model with presentday dark-matter density (in units of the critical density) $\Omega_{d}=0.25$, baryon density $\Omega_{b}=0.05$, cosmological constant $\Omega_{\Lambda}=0.70$, Hubble parameter $H_{0}=$ $72 \mathrm{~km} \mathrm{~s}^{-1} \mathrm{Mpc}^{-1}$, and spectral index $n=1$.

In the standard $\Lambda \mathrm{CDM}$ model, the initial curvature perturbations of the Universe, presumably produced by inflation or some inflationlike mechanism, are adiabatic (perturbations in the total density but not the relative density between species) and Gaussian with a nearly scale-invariant spectrum of amplitudes. These initial perturbations grow and react under the influence of gravity and other forces, with the exact nature of their behavior dependent upon the species in question. Because dark-matter particles are, by assumption, cold and collisionless, the fractional dark-matter-density perturbation $\delta_{d} \equiv \delta \rho_{d} / \rho_{d}$ can grow only under the influence of gravity. The baryonic species, being charged, are tightly coupled by Coulomb scattering to the electrons, which are themselves tightly coupled to the photons via Thomson scattering. The baryons and photons can thus be described at early times as a single baryon-photon fluid, with the photons providing most of the pressure and inertia and the baryons providing only inertia. Gravity will tend to compress this baryon-photon fluid, while the radiation pressure will support it against this compression. The result is acoustic oscillation, and the baryon density perturbation $\delta_{b} \equiv \delta \rho_{b} / \rho_{b}$ and photon density perturbation $\delta_{\gamma} \equiv \delta \rho_{\gamma} / \rho_{\gamma}$ will oscillate in time for length scales inside the horizon (on length scales larger than the horizon the pressure can have no effect). At early times, these perturbations are very small and linear perturbation theory can be applied. This allows an arbitrary density field to be decomposed into a set of independently evolving Fourier modes, labeled by a wave number $k$. Figure 1 shows the growth of a dark-matter 


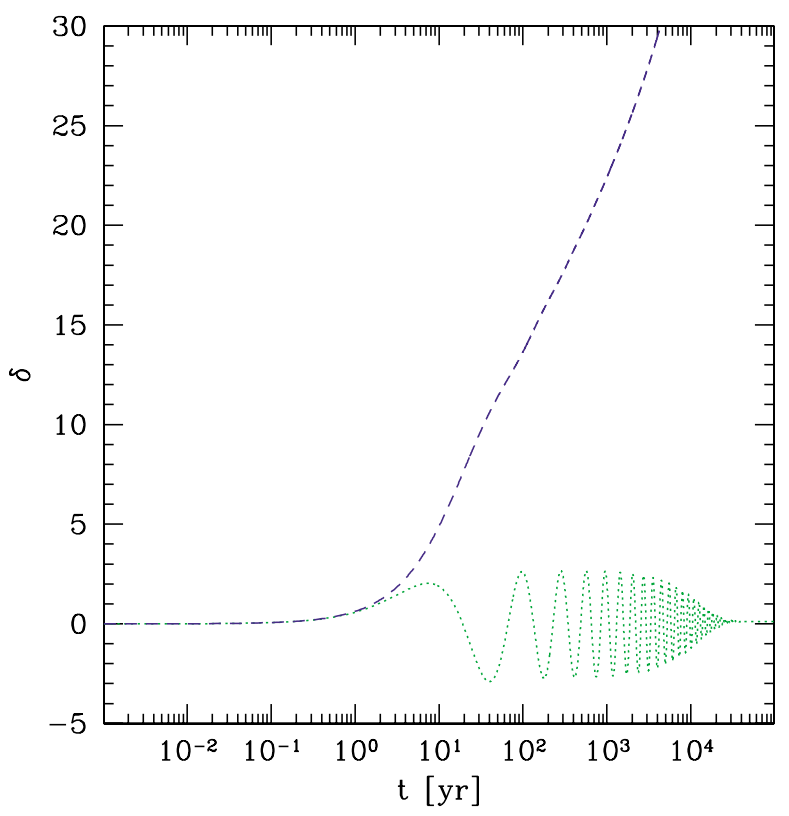

FIG. 1 (color online). The evolution of the comoving wave number $k=3.0 \mathrm{Mpc}^{-1}$ density perturbations in the early Universe for dark matter (dashed line) and for baryons (dotted line). The dark-matter perturbation always grows under the influence of gravity while the baryonic perturbation oscillates due to a competition between gravity and the photon pressure.

density perturbation under the influence of gravity, and the oscillatory behavior of the baryon perturbation for the same wave number.

We choose to work in the synchronous gauge where the time slicing is fixed to surfaces of constant proper time so that particle decays proceed everywhere at the same rate. In the synchronous gauge, the standard linearized evolution equations for perturbations in Fourier space are (e.g., [7])

$$
\begin{gathered}
\dot{\boldsymbol{\delta}}_{d}=-\theta_{d}-\frac{1}{2} \dot{h}, \quad \dot{\theta}_{d}=-\frac{\dot{a}}{a} \theta_{d}, \\
\dot{\boldsymbol{\delta}}_{b}=-\theta_{b}-\frac{1}{2} \dot{h}, \\
\dot{\theta}_{b}=-\frac{\dot{a}}{a} \theta_{b}+c_{s}^{2} k^{2} \delta_{b}+\frac{4 \rho_{\gamma}}{3 \rho_{b}} a n_{e} \sigma_{T}\left(\theta_{\gamma}-\theta_{b}\right), \\
\dot{\boldsymbol{\delta}}_{\gamma}=-\frac{4}{3} \theta_{\gamma}-\frac{2}{3} \dot{h},
\end{gathered}
$$

and

$$
\dot{\theta}_{\gamma}=k^{2}\left(\frac{1}{4} \delta_{\gamma}-\Theta_{\gamma}\right)+a n_{e} \sigma_{T}\left(\theta_{b}-\theta_{\gamma}\right),
$$

where $\theta_{b}, \theta_{d}$, and $\theta_{\gamma}$ are the divergence of the baryon, dark matter, and photon fluid velocities, respectively, and an overdot represents a derivative with respect to the conformal time $\eta$. Here $h$ is the trace of the spatial metric perturbations $h_{i j}$. Its evolution is described by the linearized Einstein equations, which close this system of linearized equations. The last terms on the right-hand sides of Eqs. (3) and (5) account for Thomson scattering between baryons and photons, and are responsible for keeping them tightly coupled in the early Universe. In these equations $\sigma_{T}$ is the Thomson cross section, $n_{e}$ is the electron number density, and $c_{s}$ is the intrinsic sound speed of the baryons. During tight coupling, the second moment $\Theta_{\gamma}$ of the photon distribution and other higher moments can be neglected, and the radiation can reliably be given the fluid description described above.

We now show how Eqs. (1)-(5) are modified by the decay of a long-lived metastable charged particle to dark matter in the early Universe. We assume that the decay is of the form $q^{ \pm} \rightarrow \ell^{ \pm} d$, so the decay of each charged particle $q^{ \pm}$produces a dark-matter particle $d$ and a charged lepton $\ell^{ \pm}$. Denoting the decaying charged component by the subscript $q$, the background density $\rho_{q}$ evolves according to the equation

$$
\dot{\rho}_{q}=-3 \frac{\dot{a}}{a} \rho_{q}-\frac{a}{\tau} \rho_{q},
$$

where $\tau$ is the lifetime of $q^{ \pm}$. The first term just accounts for the normal $a^{-3}$ scaling of nonrelativistic matter in an expanding universe, while the second leads to the expected exponential decay of the comoving density. For the dark matter we have

$$
\dot{\rho}_{d}=-3 \frac{\dot{a}}{a} \rho_{d}+\lambda \frac{a}{\tau} \rho_{q},
$$

where $\lambda=m_{d} / m_{q}$ is the ratio of the masses of the darkmatter particle to the charged particle. The energy density in photons evolves according to

$$
\dot{\rho}_{\gamma}=-4 \frac{\dot{a}}{a} \rho_{\gamma}+(1-\lambda) \frac{a}{\tau} \rho_{q} .
$$

This last equation follows from the assumption that the produced lepton initiates an electromagnetic cascade which rapidly (compared to the expansion time scale) thermalizes with the photon distribution. In practice, the last term on the right-hand side of Eq. (8) is negligibly small because the decay takes place during the radiation dominated era when $\rho_{\gamma} \gg \rho_{q}$. Furthermore, limits on the magnitude of $\mu$ distortions to the blackbody spectrum of the CMB constrain $|1-\lambda|$ to be a small number, as we discuss below.

Using covariant generalizations of Eqs. (6)-(8), we can derive how Eqs. (1)-(5) are modified by the transfer of energy and momentum from the $q$ component to the dark matter during the decay process. Since the charged $q$ component and the baryons are tightly coupled via Coulomb scattering, they share a common velocity $\theta_{\beta}=$ $\theta_{b}=\theta_{q}$. This makes it useful to describe them in terms of a total charged-species component with energy density $\rho_{\beta}=\rho_{b}+\rho_{q}$, which we denote here by the subscript $\beta$. Because in the synchronous gauge the decay proceeds everywhere at the same rate, this description is even 
more useful as $\delta_{\beta}=\delta_{b}=\delta_{q}$ is maintained at all times for adiabatic initial conditions. In terms of these $\beta$ variables, then, we have

$$
\begin{gathered}
\dot{\delta}_{d}=-\theta_{d}-\frac{1}{2} \dot{h}+\lambda \frac{\rho_{q}}{\rho_{d}} \frac{a}{\tau}\left(\delta_{\beta}-\delta_{d}\right), \\
\dot{\theta}_{d}=-\frac{\dot{a}}{a} \theta_{d}+\lambda \frac{\rho_{q}}{\rho_{d}} \frac{a}{\tau}\left(\theta_{\beta}-\theta_{d}\right), \\
\dot{\delta}_{\beta}=-\theta_{\beta}-\frac{1}{2} \dot{h}, \\
\dot{\theta}_{\beta}=-\frac{\dot{a}}{a} \theta_{\beta}+c_{s}^{2} k^{2} \delta_{\beta}+\frac{4 \rho_{\gamma}}{3 \rho_{\beta}} a n_{e} \sigma_{T}\left(\theta_{\gamma}-\theta_{\beta}\right), \\
\dot{\delta}_{\gamma}=-\frac{4}{3} \theta_{\gamma}-\frac{2}{3} \dot{h}+(1-\lambda) \frac{\rho_{q}}{\rho_{\gamma}} \frac{a}{\tau}\left(\delta_{\beta}-\delta_{\gamma}\right),
\end{gathered}
$$

and

$$
\begin{aligned}
\dot{\theta}_{\gamma}= & k^{2}\left(\frac{1}{4} \delta_{\gamma}-\Theta_{\gamma}\right)+a n_{e} \sigma_{T}\left(\theta_{\beta}-\theta_{\gamma}\right) \\
& +(1-\lambda) \frac{\rho_{q}}{\rho_{\gamma}} \frac{a}{\tau}\left(\frac{3}{4} \theta_{\beta}-\theta_{\gamma}\right) .
\end{aligned}
$$

We now describe how small-scale modes that enter the horizon prior to decay are suppressed relative to those modes that enter the horizon after decay. Because of the Thomson collision terms, the $\beta$ component and the photons will be tightly coupled as a $\beta$-photon fluid at early times and this fluid will support acoustic oscillations. Furthermore, Eqs. (9) and (10) show that the dark-matter perturbations are strongly sourced by the perturbations of the $\beta$ component prior to decay, when the ratio $\rho_{q} / \rho_{d}$ is large. Dark-matter modes that enter the horizon prior to decay will thus track the oscillations of the $\beta$-photon fluid rather than simply growing under the influence of gravity. After decay, when the ratio $\rho_{q} / \rho_{d}$ is small, the source term shuts off and dark-matter modes that enter the horizon undergo the standard growing evolution. In Fig. 2, we follow the evolution of the dark-matter perturbations through the epoch of decay. We modified CMBFAST [8] to carry out these calculations.

In order to suppress power on subgalactic scales, the decay lifetime must be roughly the age of the Universe when the mass enclosed in the Hubble volume is equal to a galaxy mass; this occurs when $\tau \sim$ yr. In Fig. 3, we plot the linear power spectrum of matter density fluctuations at the present day for a charged-particle lifetime $\tau=$ $3.5 \mathrm{yr}$ assuming a scale-invariant primordial power spectrum. We see that power is suppressed on scales smaller than $k^{-1} \sim 0.3 \mathrm{Mpc}$ relative to the standard $\Lambda \mathrm{CDM}$ power spectrum. Suppression of power on these length scales reduces the expected number of subgalactic halos, bringing the predictions in line with observation [5] without violating constraints from the Lyman-alpha forest [9]. It leaves the shapes of galactic halo density profiles essentially unchanged [10]. Of course, the model reproduces the successes of the standard $\Lambda \mathrm{CDM}$ model on larger scales and in the CMB.

The requirements of the charged-particle species are that it have a comoving mass density equal to the darkmatter density today and have a lifetime of $\tau \sim 3.5 \mathrm{yr}$. In order to satisfy the constraint to the CMB chemical potential [11], the fractional mass difference between the charged and neutral particles must be $\Delta m / m<3.6 \times$ $10^{-3}$, and in order for the decay to be allowed kinematically the mass difference must be greater than the electron mass. One possibility is the superweakly interacting massive particle scenario of Ref. [12] in which a charged particle may decay to an exclusively gravitationally interacting particle. For example, in supersymmetric models, the decay of a selectron to an electron and gravitino $\tilde{e} \rightarrow e \tilde{G}$ with $m_{\tilde{e}} \approx m_{\tilde{G}}>122 \mathrm{TeV}$ would satisfy these constraints, as would the decay of a Kaluza-Klein (KK) electron to an electron and KK graviton $e^{1} \rightarrow e G^{1}$ with

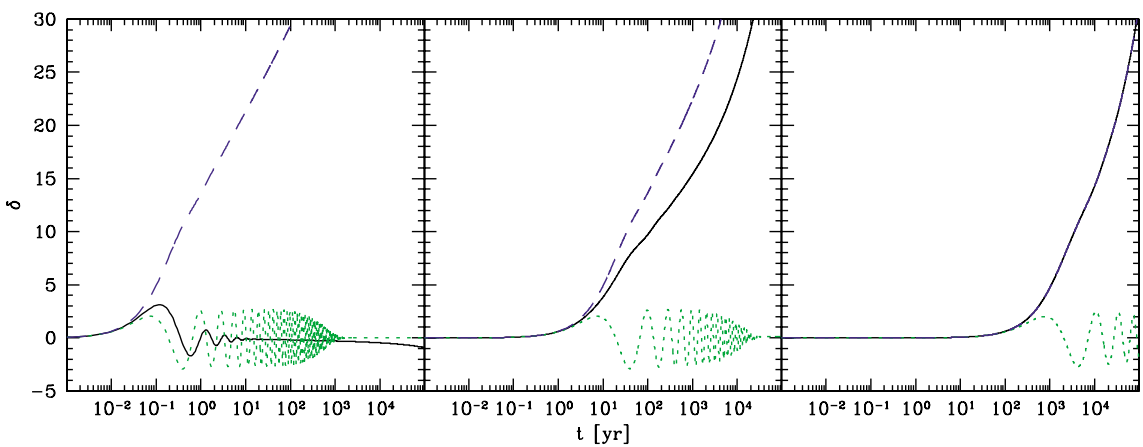

FIG. 2 (color online). The evolution of the comoving wave number $k=30.0 \mathrm{Mpc}^{-1}$ (left panel), $k=3.0 \mathrm{Mpc}-1$ (center panel), and $k=0.3 \mathrm{Mpc}^{-1}$ (right panel) density perturbations in the early Universe for dark matter in the $\Lambda$ CDM model (dashed line) and in the model with $\tau=3.5 \mathrm{yr}$ (solid line). The $\beta$ perturbation is represented by the dotted line. Because of being sourced by the low amplitude $\beta$ perturbations at early times, the dark matter perturbation in the model with a decaying charged component is suppressed relative to the standard $\Lambda \mathrm{CDM}$ case for $k=3.0 \mathrm{Mpc}^{-1}$. For $k \gg 3.0 \mathrm{Mpc}^{-1}$ (very small scales), $\delta_{d}$ tracks the oscillations in $\delta_{\beta}$ before decay, while for $k \ll 3.0 \mathrm{Mpc}^{-1}$ (large scales) $\delta_{d}$ follows the standard growing evolution. 


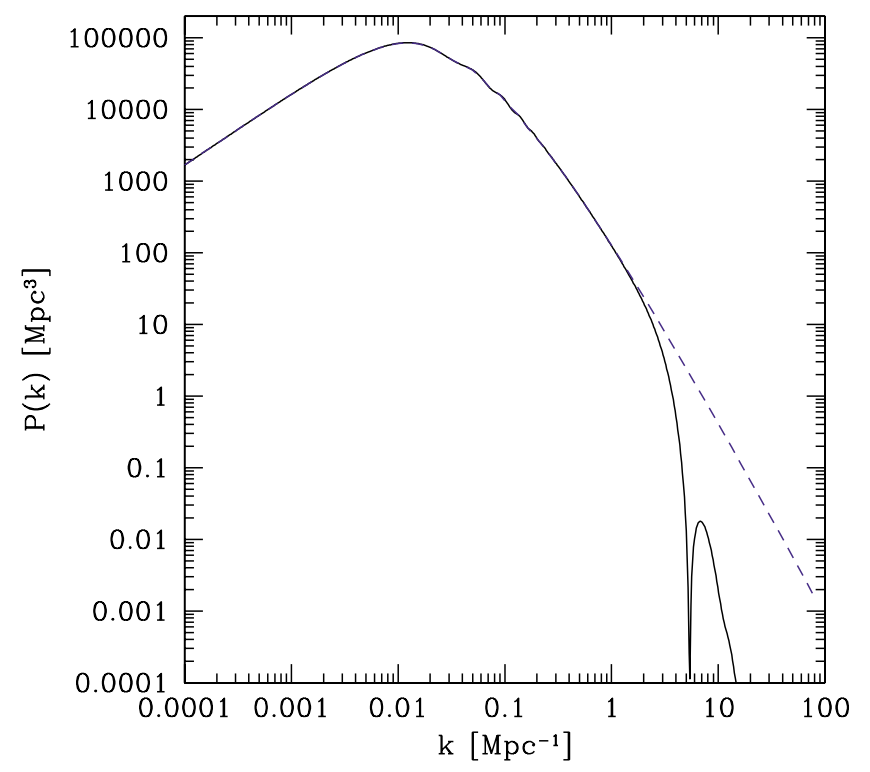

FIG. 3 (color online). The linear-theory power spectrum of matter density fluctuations in the standard $\Lambda$ CDM model (dashed line), and in the charged decay to dark matter model (solid line) with $\tau=3.5 \mathrm{yr}$. The charged decay model matches the standard $\Lambda \mathrm{CDM}$ model on length scales larger than $0.3 \mathrm{Mpc}$, but power drops off sharply below $0.3 \mathrm{Mpc}$.

$m_{e^{1}} \approx m_{G^{1}}>72 \mathrm{TeV}$ in the case of the single universal extra dimension KK model discussed in Refs. [12,13]. Such masses are close to the unitary bound for thermal production [14], but might be accommodated through nonthermal mechanisms or if the next-to-lightest partner is a squark which might then interact more strongly and thus evade this bound. There may also be viable scenarios involving nearly degenerate charged and neutral higgsinos.

It should be noted that the recent Wilkinson microwave anisotropy probe evidence for early star formation [15] argues against the suppression of small-scale power, but these results are not yet conclusive. If it does turn out that traditional astrophysical mechanisms can explain the dearth of dwarf galaxies, then our arguments can be turned around to provide constraints to an otherwise inaccessible region of the parameter space for decaying dark matter [16]. Finally, if the mechanism we propose here is realized in nature, then the dearth of small-scale power, along with the detection of a nonzero CMB chemical potential, would be a powerful probe of the particle spectrum of the new physics responsible for dark matter.
K. S. acknowledges the support of the Canadian NSERC. This work was supported in part by NASA NAG5-9821 and DoE DE-FG03-92-ER40701.

*Electronic address: ksigurds@tapir.caltech.edu

${ }^{\dagger}$ Electronic address: kamion@tapir.caltech.edu

[1] P. de Bernardis et al., Nature (London) 404, 955 (2000); S. Hanany et al., Astrophys. J. Lett. 545, L5 (2000); N.W. Halverson et al., Astrophys. J. 568, 38 (2002); B. S. Mason et al., Astrophys. J. 591, 540 (2003); A. Benoit et al., Astron. Astrophys. 399, L25 (2003); J. H. Goldstein et al., Astrophys. J. 599, 773 (2003); D. N. Spergel et al., Astrophys. J. Suppl. Ser. 148, 175 (2003).

[2] J. A. Peacock et al., Nature (London) 410, 169 (2001); W. J. Percival et al., Mon. Not. R. Astron. Soc. 327, 1297 (2001); M. Tegmark et al., astro-ph/0310725.

[3] G. Kauffmann, S. D. M. White, and B. Guiderdoni, Mon. Not. R. Astron. Soc. 264, 201 (1993); A. A. Klypin et al., Astrophys. J. 522, 82 (1999); B. Moore et al., Astrophys. J. Lett. 524, L19 (1999).

[4] A. J. Benson et al., Mon. Not. R. Astron. Soc. 333, 177 (2002); R. S. Somerville, Astrophys. J. Lett. 572, L23 (2002); L. Verde, S. P. Oh, and R. Jimenez, Mon. Not. R. Astron. Soc. 336, 541 (2002).

[5] M. Kamionkowski and A. R. Liddle, Phys. Rev. Lett. 84, 4525 (2000).

[6] C. Boehm, P. Fayet, and R. Schaeffer, Phys. Lett. B 518, 8 (2001); X. Chen, M. Kamionkowski, and X. Zhang, Phys. Rev. D 64, 021302 (2001); X. Chen, S. Hannestad, and R. J. Scherrer, Phys. Rev. D 65, 123515 (2002); C. Boehm et al., astro-ph/0309652; D. N. Spergel and P. J. Steinhardt, Phys. Rev. Lett. 84, 3760 (2000).

[7] C.-P. Ma and E. Bertschinger, Astrophys. J. 455, 7 (1995).

[8] U. Seljak and M. Zaldarriaga, Astrophys. J. 469, 437 (1996).

[9] M. White and R. A.C. Croft, Astrophys. J. 539, 497 (2000).

[10] B. Moore et al., Mon. Not. R. Astron. Soc. 310, 1147 (1999).

[11] D. J. Fixsen et al., Astrophys. J. 473, 576 (1996).

[12] J. L. Feng, A. Rajaraman, and F. Takayama, Phys. Rev. Lett. 91, 011302 (2003); Phys. Rev. D 68, 063504 (2003).

[13] T. Appelquist, H.-C. Cheng, and B. A. Dobrescu, Phys. Rev. D 64, 035002 (2001).

[14] K. Griest and M. Kamionkowski, Phys. Rev. Lett. 64, 615 (1990).

[15] A. Kogut et al., Astrophys. J. Suppl. Ser. 148, 161 (2003).

[16] K. Sigurdson and M. Kamionkowski (to be published). 